\title{
Telepsychology: Public Speaking Fear Treatment on the Internet
}

\author{
C. BOTELLA, Ph.D., ${ }^{1}$ R. BAÑOS, Ph.D ${ }^{2}$ V. GUILLÉN, ${ }^{1}$ C. PERPIÑA, Ph.D., ${ }^{2}$ \\ M. ALCAÑIZ, Ph.D., ${ }^{3}$ and A. PONS ${ }^{4}$
}

\section{INTRODUCTION}

$\mathbf{U}$ NTIL VERY RECENTLY, the possibility of starting real telemedicine or telepsychology systems was restricted by the cost of the equipment and by the lack of adequate infrastructures. Nonetheless, the technological advances we are witnessing at present have allowed the publication of a series of pioneering works with good and promising results. The analysis of what is happening in the field of telepsychology points to an important development to come in the following years.

This work presents a telepsychology system to the treatment of public speaking fear. The system is composed of: (a) an assessment protocol that gives the patient a diagnosis of his/her problem, including the interference it is causing him/her, its severity, and the degree of fear and avoidance it is producing; (b) a structured treatment protocol, organized in separate blocks reflecting the patient's progress; in this way, it is possible to ensure that the patient does not skip any step in the treatment (something quite common in the traditional self-help manuals), gaining more control over the process; and (c) an outcome protocol that assesses treatment effectiveness, not only at its end, but also at every intermediate step. It is the first work in a promising research line that examines the possibility of using the Internet for the treatment of psychological dis- orders. If the program proposed here achieves the necessary effectiveness and soundness, the range of psychological problems to which the program could be applied will broaden in the near future.

\section{BACKGROUND}

Virtual reality as a therapeutic tool in clinical psychology

Two years ago, Wiederhold ${ }^{1}$ stated that we were entering the second wave of virtual reality (VR) applications. In this author's opinion, the first wave would refer to all those applications involving high-cost systems, such as the Silicon Graphics platforms. At that moment, the possibilities for action were clearly limited by the difficulty of developing applications that could operate in low-cost systems, such as personal computers. That is why the advances achieved in the binomial cost-benefit ${ }^{2}$ have meant an important step toward the crystallization and expansion of this second wave.

In the field of psychological treatments, this "new way of therapy" implies several advantages for the therapeutic setting. ${ }^{3,4}$ VR offers a very special "reality" with a great utility as many of the aspects traditionally considered as core for the good evolvement of the therapeutic process, meet in VR.

\footnotetext{
${ }^{1}$ Universidad Jaume I, Castellón, Spain.

${ }^{2}$ Universidad de Valencia, Valencia, Spain.

${ }^{3}$ Universidad Politécnica de Valencia, Valencia, Spain.

${ }^{4}$ Universidad Cardenal Herrera, CEU Valencia, Spain.
} 
1. Therapy as a special and protected environment, where the patient can start to explore and act, constitutes one of the "chief ingredients of therapy." 5,6

2. Role performance is a basic strategy used in every therapeutic school; in VR, it implies to act without feeling threatened, neither by the world, others, and even oneself.

3. VR has an outstanding value in "individualizing" therapy, since (at least, theoretically) the feared environments can be adjusted and tailored to the specific difficulties of every single patient.

4. VR also fosters the generalization of the achievements, as it is possible to design different scenarios where the patient can practice his/her recently acquired skills.

5. VR allows a greater level of control over time because it is not necessary to wait for the events to occur.

6. The same holds true regarding the control over space: many feared situations are not easily accessible in the real world, but VR goes beyond reality.

7. Apart from the possibility of self-training, the person can work in the situation at his/her own pace, and as many times as he/she may need, in order to achieve a real over-learning. ${ }^{3,4}$

All these reasons stand up our understanding of VR as an intermediate step between the therapist's consultation room (a totally protected environment) and the real world (so threatening). And this mediating step turns out to be a primary step, as many patients refuse to give it in their whole lives.

It is no wonder, thus, that numerous VR applications had sprung in the field of psychological treatments, proving their efficacy and clinical utility, being the treatment of phobias the most paradigmatic. It is necessary to mention the first softwares designed by Rothbaum and North's group to the treatment of acrophobia, $^{7,8,9}$ agoraphobia ${ }^{10,11}$ spider phobia, ${ }^{12}$ or flying phobia. ${ }^{13}$ Their results reveal the effectiveness of VR to the treatment of the mentioned disorders. ${ }^{14}$ Our group has also made its contribution with a VR software for the treatment of claustrophobia and another for the treatment of flying phobia. ${ }^{15}$ The former has been already tested, and has yielded sound results in the clinical population suffering from claustrophobia. ${ }^{4}$ We have observed the generalization of the outcome obtained to other phobic behaviors that were not the target of treatment. ${ }^{16}$ The latter is currently being run in a clinical sample. Furthermore, the VR strategies not only have proved their efficacy in specific problems such as phobias, but also in the treatment of other more complex disorders such as eating disorders with equally promising results. ${ }^{17,18}$

It is necessary, though, to keep working in this line so the hardware and software systems become "friendlier" and more accessible. Nonetheless, after analyzing the developments and achievements attained in the last decade it is no doubt that, in short, VR will be within everybody's reach.

\section{Self-help}

Self-help material can be defined as any means (written, recorded, etc.) whose content is a treatment program (or part of it) that may be self-administered by patients with or without the therapist's guidance. ${ }^{19}$ Glasgow and Rosen's revisions ${ }^{19,20}$ of the usage of self-help procedures in behavior therapy reveal their efficacy and are optimistic about its utility in a wide array of psychological problems (phobias, obesity, sexual dysfunctions, assertiveness, child behavioral problems, study techniques, tobacco problems, etc.). Nonetheless, these authors insist on the need of validating any self-help procedure in clinical populations before it could be applied to the general population and of developing cost-benefit and effectiveness indices before deciding about the convenience of applicating the program. They also add a series of proposals regarding such a validation process.

Rosen, in a later revision, ${ }^{21}$ remarks the spread of this kind of material and also insists on the need of conducting thorough research in this area because an indiscriminate and unjustified use of certain self-help material might even exacerbate the problem. For instance, a person may make an erroneous diagnosis of his/her problem and self-apply an inadequate treatment that not only does not alleviate his/her trouble, but also worsen it. It also may be that he/she misunderstands the instructions 
and guidelines and applies the treatment incorrectly, or for too little a time. Whatever the error, it can lead to a failure that can be misinterpreted by the person and, thus, deplete his/her trust in psychological treatments.

Besides the cautions raised by Rosen, ${ }^{21}$ another important aspect in our opinion is the fact that the information is offered in one go, that is, without taking precautions regarding whether every step along the therapeutic process is given in the appropriate way. For that very reason, one of the main aspects of the program presented here is to shape a series of controls and barriers thought to ensure that diagnosis and treatment are achieved appropriately.

In the last revision of this issue, ${ }^{22}$ Gould and Clum reached some general conclusions about the efficacy of these methods in the treatment of psychological problems. Such conclusions are quite optimistic and leave the door open to the design of self-help material whenever the adequate and essential methodological rigor is taken into account in order to keep progressing in any aspect of therapy.

In view of all this, it seems sensible to think of methods that allow psychological assistance in a different way, provided that the results on therapeutic effectiveness and on cost-benefit conditions are encouraging. During the last years, our group has focused on this kind of problem and has put to test the possibility of shortening the contact between patient and therapist by means of structured self-help material. The outcome on efficacy was similar in both the long and the short programs, ${ }^{23}$ independent of the status on medication of patients. ${ }^{24}$ We believe that the successes obtained in this field will imply an important step forward in the removal of restrains such as the limited accessibility and/or the high cost of the cognitive-behavioral interventions. According to the National Institute of Health, ${ }^{25}$ those are the main reasons why many patients do not benefit from the current treatment alternatives

\section{TELEPSYCHOLOGY (TELE-MENTAL-HEALTH)}

Telehealth $(\mathrm{TH})$ is the standard term chosen by the Standing Committee of Family and
Community Affairs"26 to refer to the "remote provision of health." TH may be defined as "any application that implies carrying out activities related to health (i.e., a health, education, and/or information service) remotely, using computers and telecommunications" 27 (p. 6).

Chief characteristics of $\mathrm{TH}$ systems are the geographical separation between the person that provides the service and the user, and the use of telecommunication technologies with the purpose of facilitating such an interaction. ${ }^{28}$ Within the wide framework of $\mathrm{TH}$, several related disciplines such as Telemedicine, Telepsychiatry, and Telepsychology have appeared. The technologies implied in these disciplines are videoconferences, telephones, computers, Internet, fax, radio, and television. Telepsychiatry or Telepsychology have been defined as "the use of telecommunication technologies to put in contact patients with health professionals in order to accomplish tasks such as medical or psychological diagnoses, education, treatment, consultations, transmission and storage of the patients' medical stories and data, research and other activities related to the provision of health care." 29 In short, we are talking about providing appropriate health assistance in remote areas where there are not specialized staff and/or facilities.

One of the first works undertaken in the field of telemedicine implied the use of a "long distance" communication between a Medical Department and a State Hospital with the purpose of making interconsultations. ${ }^{30}$ From the start of experiences such as this, the interest toward the possible use of the newest technological advances in telecommunications as a means of giving services through the information highways has grown rapidly. So much so that there are already programs that have wholly become part of the mental health devices in a particular area. The telepsychiatry system in Adelaida (South Australia) that has worked successfully since 1994 is an example of such programs. ${ }^{31}$

In spite of the above, the real possibility of starting telemedicine or telepsychology systems was very restricted until recently because of the cost of the equipment and the lack of adequate infrastructures regarding telecommunications, of programs specifically designed for 
this domain, and of protocols and criteria for their use. Furthermore, evidence of cost-benefit and degree of acceptance by the potential users were also lacking. Even though real efforts were made to solve these problems, there were technical constraints due to the rapidity of the net and the absence of technology able to compress audio/video material in real time. Nonetheless, technological advances are making it feasible to devise $\mathrm{TH}$ programs. It also creates an outstanding synergy among the initiatives to amend the heath services and the technological advances that support telemedicine. ${ }^{32}$ All this has allowed the emergence of several works in this field. For instance, ${ }^{33}$ digital technology has been used to provide services to chronic mental patients in isolated communities; $^{34}$ the possibility of using the phone and e-mail to diagnose and provide counseling to young psychiatric populations has been tested. Regarding diagnostic reliability, ${ }^{35}$ it has been concluded that the psychiatric assessment of a patient through videoconferencing is as reliable as the face-to-face consultations. It has also been proved by several studies $^{32,36-38}$ that this new way of providing mental health care is well accepted by the users. Most users report a degree of satisfaction for the remote assistance similar to that for the traditional consultation.

All these new developments in the domain of mental health reveal the need for redefining the provision models of basic services (such as assessment and treatment) so that they include: health care at home and telepsychology and telepsychiatry, practical guidelines and basic ways of proceeding, use of paraprofessionals, management of cases, harm prevention, and health promotion. ${ }^{39}$ In short, redefinitions of access to these services and how they are provided. We are, therefore, facing a new era that, in our opinion, may entail a deeper revolution of basic concepts and ways of functioning in the mental health arena than the one that started the community of psychiatry and psychology. ${ }^{40}$

However, now there are no controlled selfhelp programs designed for its application via the Internet, that is, using the net as a new way of making self-help feasible. As far as we know, most of the available strategies assume the use of e-mail, chats, or videoconferences. For in- stance, self-help groups or forums have been organized, and participants can communicate and exchange experiences and information. There are also lists centered on the possibilities of receiving online therapy, and web pages where certain professionals offer their telepsychological services. A growing interest on telepsychology by some authors has been observed as well (see, for instance, http://www. rider.edu/users/suler/psycyber/therapy.html .). Several specialized articles have been published in prestigious journals, ${ }^{41}$ and guides to trace the existing online mental health resources have appeared $^{42}$ and are continually updated at http://www.insidemh.com. In light of what has been said, it is no wonder that the Monitor on Psychology (April) is a monograph on psychology and Internet.

If we focus on the domain of online treatments, a quite frequent strategy is to provide information on particular disorders and the existing therapeutic possibilities. An example of it can be found in http://www.mentalhelp.net/guide/pro33. htm, within the TAPIR ("The Anxiety Panic Internet Resource") frame. The TAPIR offers a possibility of self-help for the treatment of anxiety disorders, but such help is limited to giving relevant information to the user (for instance, guidelines on how to recognize the characteristics of the disorder, information on the treatments currently available, and how to get in touch with the professionals or centers that apply them). However, regarding the kind of selfhelp that involves self-application of treatments, TAPIR only provides a list of general rules to follow in case of a panic attack and some exercises for stress reduction. Therefore, TAPIR is an innovative first step, but insufficient. On one hand, it is simple and fragmented compared with the self-help books dealing with this subject. ${ }^{43}$ On the other hand, it suffers from the general problems encountered in self-help, as it does not ensure whether or not the person can make the correct diagnostic of his/her problem, and it does not control the way in which the person applies the treatment.

\section{THE TREATMENT PROGRAM}

The treatment program presented in this work is a telepsychology system that uses the 
net as the medium for its application. It is designed to guide the person along the whole therapeutic process. The system is composed of: (a) an assessment protocol that gives to the person a diagnosis of his/her problem, including the interference it is causing him/her, its severity, and the degree of fear and avoidance it is producing; (b) a structured treatment protocol, organized in separate blocks reflecting the person's progress; in this way, it is possible to ensure that he/she does not skip any step in the treatment, gaining more control over the process; and (c) an outcome protocol that assesses treatment effectiveness, not only at its end, but also at every intermediate step.

Given that it is a new system and that there is barely any experience in this domain, public speaking phobia was chosen as the problem targeted by the program. This syndrome does not entail the difficulties of other more complex a risky disorders such as major depression disorder or posttraumatic stress disorder.

\section{Psychological aspects}

The system is designed to be able to operate efficaciously from a cost-benefit perspective via the Internet. It pursuits, therefore, a twofold purpose: to be applicable, step-bystep, by the patient and to provide all the help that the person might need through the whole process.

Reliance. A primary aspect to be considered is the reliance of the user regarding the guarantees that the system offers from a psychological and professional perspective. The key aspects that the user may wonder about would be: Do the persons who have designed the system have any knowledge on psychology? Do they know anything about my problem? What kind of treatment is applied? Is it clinically validated? What about confidentiality? Thus, the system starts trying to offer to the potential user guarantees in those key aspects:

- Who are we? The system lists about each one of the members of the research and therapy team and their credentials, and offers objective ways of contrasting that information.
- Which problem? The problem that the system targets is explained: public speaking fear.

- Which treatment? The rationale for the treatment is given, and the person is informed about its proven effectiveness in tackling the problem.

- Who knows about it? This point addresses the safety of the system in data protection.

- How to use it? Once the previous issues are clarified, the system explains to the user its logic, that is, the way it can be used. Several aspects such as the mode of carrying the self-application out, potential problems that can arise, and how to solve them are approached.

Rapport and empathy. Then, the system introduces itself. It tells the user its name ("Talk to me") and its purpose: to be there to guide and offer help. Such help means to protect the person at any point (by not allowing him/her to go on if he/she is not ready to do so), and to ensure an adequate application. "Talk to me" explains that it will make periodical assessments throughout the process and, depending on the results, will advise which is the best thing to do next.

Assessment. "Talk to me" presents a series of questionnaires that the user has to fill out. Then, it offers a brief psychological profile regarding the fear of public speaking. Depending on the scores, "Talk to me" informs to the person whether: (a) he/she will benefit of the procedure or (b) he/she should seek another type of specialized help as there are data to suggest the inconvenience of proceeding.

Next, "Talk to me" tracks down the possible target behaviors for the user, as well as the degree of fear, avoidance, and worry experienced by the person related to the potential negative evaluation he/she is afraid of suffering if he/she gets involved in each one of the target behaviors. The achievement of the target behaviors would mean that the problem the person presented has been overcome. "Talk to me" saves all the information gathered through the assessment in order to, on one hand, shape the treatment program the person needs and, on the other, assess the progress, stagnation, or 
worsening of the public speaking fear along the therapy process.

Treatment. "Talk to me" informs that treatment consists of a series of components: psychoeducation, exposure, attentional focus, and cognitive restructuring. Psychoeducation shows with detail the main aspects and the rationale for the remaining components. After each presentation, "Talk to me" makes sure that the person has understood and assimilated the information by means of several self-assessment questions. In case these questions were not correctly answered, the person would be asked to read again specific parts of the information.

"Talk to me" has several basic scenarios typically feared by the persons afraid of public speaking: (a) the classroom; (b) the congress; (c) the oral exam; (d) the oral presentation of a project; (e) the congratulation-lunch speech; (f) the listening group of friends. Each scenario has a series of modulators that allow for a hierarchy within situations for each person. The system will show one or more scenarios in a determined order that will depend on the results of the assessment.

"Talk to me" tells the user what he/she has to do when confronted with the feared scenario. At the beginning of each session, the person indicates his/her fear, avoidance, and worry about negative evaluation in the situation that he/she is going to face. Then, it presents the corresponding scenario (according to person and session). Every 5 minutes it asks for rates of fear, avoidance, and worry, and reminds to the user that he/she should not leave the exposure unless anxiety diminishes. At the end of the session, it shows the graphics of the progress experienced by the person along the session and congratulates him/her for their efforts. The same process takes place in the following sessions until the person successfully overcomes all his/her target behaviors.

Finally, it should be noted that the system has been designed to be self-applied. The user may address any additional question, doubt, or comment to any of our therapists via e-mail, phone, or fax. We are currently testing the program with a clinical sample of university students suffering from public speaking fear. We will gather the results in the level of fear, avoidance, and worry, and the generalization to reallife public speaking situations.

\section{Technical aspects}

Networked telepsychology implementation objectives. The adaptation of the program to the Internet involved the application of a range of available technologies. At an application development level, a number of implementation aspects were considered independently.

1. Firstly, the environment is presented to the user as a set of stimuli related to the pathology treated. For instance, an auditorium setting with posed questions could be provided to treat public speaking fear. These are controlled simulations presented to the user by means of complementary techniques: interactive video downloaded from the net in real-time using serialization and buffering; $3 \mathrm{D}$ virtual environments that have design and hardware requirements dependent on the complexity of the stimuli; or descriptions of texts and situations to which a common web implementation or a more advanced multimedia presentation could be applied.

2. Secondly, a control of patient activity was provided. The aim was not to present the patient with a sequence of unordered stimuli, but to follow a controlled flow decided and supervised by the system. Therefore, the access to the different sessions is controlled and assessed achieving an effective feedback of the process of the person through tests or other instruments decided by the psychologist. This task was subdivided into two main topics:

I. Set-up of a patient state control system. For this purpose a set of possible status needed to be predefined in addition to the actions connected to each state. Moreover, the flow or "trajectory" of the person through the system had to be configurable according to his/her particular needs. A range of trials and tests might be needed to carry out in order to design a "graph" of the possibilities that the person had to explore. This file needed to be maintained and actualized by the system, 
either through a completely centralized control located in the remote server, or allowing the user's computer to undertake its modification and signal it to the core system.

II. In addition, the connection between the patient and the psychologist had to be granted by using a number of tools. When an automatic treatment can be provided, the treatment can be implemented as a self-adaptive system. However, in case this is not possible, the direct intervention of the psychologist must be provided by using a range of possibilities, from simple tools such as e-mail or chat to more complex elements such as videoconferences or online collaboration.

System solutions. To achieve these objectives we applied a number of technologies; one of which was the creation of stimuli: video streaming. Interactive video is a very attractive candidate for Internet uses. Video compression technology is mature enough for developing complex video and audio applications. Not only are visualization tools available, but the audio-visual material also can be adapted for real-time transmission through the Internet as a stream of data. Moreover, interesting possibilities regarding the management of events were provided. These allow marking stream locations so that events can be triggered and sent to the web page once the particular point has been reached. Thus, using DHTML, Java, and Javascript it is possible to carry out action at specific points in the video.

These possibilities can be applied to controlling the video presentation. Therefore, a threatening situation for the user can be presented allowing for an advanced degree of interactivity. The application knows the particular time in which a stimulus is presented, and the appropriate help or explanation accompanies them. On the other hand, the feasibility of managing the events allows for action on the video stream. For instance, it is possible to select the stimulus that further affects the person or to abort the process when he/she is overwhelmed.

In order to record the material, a production team composed of qualified personnel was cre- ated: actors, producer, camera operators, and technical staff. Identifying the required scenarios was required. This way, high quality videos were recorded, leading to very effective results that enhanced the person's perception of the stimuli at maximum. For the system test, we used a Mini-DV non-linear editing system and the MPEG 4-video codec to obtain the desired quality $v s . \mathrm{Kb}$ to download.

Application control (XML data model) was another technology applied to our program. The information to present had to be framed in a computer system that gave the patient control. Two main functions were required. Firstly, the patient's state had to be monitored. A personalized treatment process was created, and a file reflecting the patient status and clinical evolution had to be maintained. Secondly, a communication channel with the team of psychologists in control of the treatment was provided.

The patient control is implemented using $\mathrm{XML}$, which is a technology with large future prospects in the Internet. It allows modeling the person's status, the actions carried out, and all the complementary data in a person's file that can be transmitted from the person's computer to the control system so that a fast and flexible management of the information is granted. The main objective of this approach is the decentralization and standardization of the data for communication between the different elements composing the system. The system architecture addressed the following goals:

- To allow the local processing of the data without requiring a centralized server providing all the system intelligence.

- To provide bi-directional data flow between server and client.

- To adapt the data so that compatibility with other systems is provided, and to allow for remote data access, for example to the clinical history of the patient.

This program used architecture for telepsychology information systems, which makes extensive use of the functionality provided by XML that was applied to all data channels. A flexible information exchange structure was built upon this basis. The flow of information is dynamic, which allows for the extraction of 
a data file of a person from the network, load it onto his/her PC, work offline onto the file, and, finally, update the modification. Intelligence is hence distributed in the network and not centralized in the server. Applications can be implemented locally using the data in the way it is required, while all the data is transferred in fully compatible XML format using the word standard HTTP protocol. The large amount of work devoted to the conceptual development of standards can be applied to this XML solution; adding object-oriented functionality for further enhancement. The use of HTTP provides full compatibility and expandability. The architecture provides a large range of interconnection possibilities, and is, therefore, very flexible.

The core of the networked telepsychology application is to create a complex data model describing each of the possible states of the person, the situations to which he/she can be exposed to, and his/her behavior in these situations. At the beginning of treatment, the person can fulfill a number of tests. The evaluation of the outcome can be performed automatically with the specially designed XML model, and can be utilized to create a tree of specific situations for that particular person. This way, the system is able to determine which of the available situations are appropriate to that user and what is the correct order of presentation. Following this approach, a file containing the core information for the treatment was created and the application was afterwards constructed on the basis of this file.

In each critical point or situation the system will be asked to obtain feedback from the person, so that the system can adapt dynamically according to the needs of therapy depending on his/her evolution and reactions due to the stimuli. The aim of this structure is to eliminate the presence of the psychologist as much as possible. Thus, the psychologist can supervise the therapy process without the patient noticing it. The self-guided philosophy of the application allows for the treatment of a large number of patients by a reduced team of psychologists.

Direct interaction between psychologist and user is also provided. Traditional methods such as e-mail and chats were improved and used for this purpose. It is appropriate to provide specifically designed communication facilities that are capable of organizing the information in categories, rather than by means of a nonordered communication. The person has always the chance to establish communication with the psychologist at the proper time, but he/she does not need to open a number of applications for this purpose: The whole communication system is integrated in the application. On the other hand, the psychologist has a personal web page where he/she can send queries to the user. The different questions are categorized and ordered and the information is presented together with the rest of the person's data in an ordered fashion.

\section{FINAL THOUGHTS}

The assessment and treatment protocols presented have the advantage of "measuring out" the amount of information given according to every particular person's needs and progresses. This way, the person does not skip information or main steps to achieve improvement.

The use of Internet and VR significantly reduces the psychological treatment costs for the user, as he/she does not need the physical presence of the therapist. Furthermore, the feared environment or situation are not always easily available or accessible for exposure. When imaging exposure is used, treatment is not so effective because it depends on the person's imaging skills. VR overcomes these difficulties by creating different environments and situations without the need of moving from one's home, school, or work site. The use of virtual exposure techniques allows self-management of the virtual environment and hold on the feared scenario with a degree of control and versatility greater than a real situation would permit. This makes the person appraise the virtual exposure as less aversive than a real exposure; making him/her more comfortable in such a situation. Still, the person's experience of an important degree of dominion and overcoming of multiple aspects and variants of the feared environments creates important personal self-efficacy expectations that strengthen the treatment efficacy. 
In vivo exposure to the feared object in real environments and situations means, in many occasions, the need of exposing oneself to public settings. This means that the problem might be known by others. However, the present program guarantees total intimacy and confidentiality as it can be run in one's home or in any other place at the person's will. This can encourage the person to seek help.

Part of the convenience of developing such programs is the reduction in costs and the easiness of access. Specific phobias are psychological disorders with the highest prevalence. However, only a small percentage of persons seek help. One of the reasons is that the problem is often not highly incapacitating and the offered treatments are expensive. Furthermore, these problems were not treated in the mental health public system in the past because of the high assistance pressure of these services.

In short, the use of the Internet in the fields of clinical psychology and psychiatry would foster treatment to a higher number of persons suffering from psychological problems. This entails development of an ethical imperative of health sciences: to put knowledge within the user's reach. Patients who do not have access to either public or private mental health facilities for different reasons (living place, schedule problems, responsibilities, etc.) would have a tool that would allow the application of an effective treatment for problems without having to move. These treatments can be used at any time and at a patient's own pace.

In the 1960s, mental health disciplines experienced a revolution with the introduction of the tenets of community psychology and psychiatry as they set up the need of "going to look for the problem wherever it is, that is, in the community." It assumed the need of professionals moving to the place where they were needed in order to achieve a substantial improvement in the quality of the offered service. We believe that the start of programs such as the one presented here may accomplish this very aim by overcoming the moving of, both, professionals and users. That is why we believe that these developments will entail another "revolution" in psychology and psychiatry.

\section{REFERENCES}

1. Wiederhold, B.K. (1998). Preface. In: Riva, G., Wiederhold, B.K., and Molinari, E. (Eds.). Virtual environments in clinical psychology and neuroscience (pp. v-vi). Amsterdam: IOS Press.

2. Botella, C., Rey, A., Perpiñá, C., Baños, R., Alcañiz, M., García-Palacios, A., Villa, H., and Lozano, J.A. (1999). Differences on presence and reality judgment using a high impact workstation and a PC workstation. Cyberpsychology and Behavior, 2, 49-52.

3. Botella, C., Baños, R., Perpiñá, C., and Ballester, R. (1998). Realidad Virtual y tratamientos psicológicos (Virtual reality and psychological treatments). Análisis y Modificación de Conducta, 24, 5-26.

4. Botella, C., Baños, R., Perpiñá, C., Villa, H., Alcañiz, M., and Rey, A. (1998). Virtual reality treatment of claustrophobia: A case report. Behaviour Research and Therapy, 36, 239-246.

5. Frank, J. (1974). Persuasion and cure. Baltimore: John Hopkins Press.

6. Korchin, S., and Sands, S. (1983). Principles common to all psychotherapies. In: Walker, C.E. (Ed.). The handbook of clinical psychology (pp. 270-299). New York: Dow Jones-Irwin.

7. North, M., and North, S. (1996). Virtual reality psychotherapy. The Journal of Medicine and Virtual Reality, 1, 28-32.

8. Rothbaum, B., Hodges, L., Kooper, R., Opdyke, D., Williford, M., and North, M. (1995). Effectiveness of computer-generated (virtual reality) graded exposure in the treatment of acrophobia. American Journal of Psychiatry, 152, 626-628.

9. Rothbaum, B., Hodges, L., Kooper, R., Opdyke, D., Williford, M., and North, M. (1995). Virtual reality graded exposure in the treatment of acrophobia: A case report. Behavior Therapy, 26, 547-554.

10. North, M., and North, S. (1994). Virtual environments and psychological disorders. Electric Journal of Virtual Culture, 2, 25-34.

11. North, M., North, S., and Coble, J.R. (1997). Effectiveness of virtual environment desensitization in the treatment of agoraphobia. Online document: http:// www.ijvr.uccs.edu/north.htm.

12. Carlin, A., Hoffman, H., and Weghorst, S. (1997). Virtual reality and tactile augmentation in the treatment of spider phobia: A case report. Behaviour Research and Therapy, 35, 153-158.

13. Rothbaum, B., Hodges, L., Watson, B., Kessler, G., and Opdyke, D. (1996). Virtual reality exposure therapy in the treatment of fear of flying: A case report. Behaviour Research and Therapy, 34, 477-481.

14. Strickland, D., Hodges, L., North, M., and Weghorst, S. (1997). Overcoming phobias by virtual exposure. Communications of the ACM, 40, 34-39.

15. Baños, R., Botella, C., and Perpiñá, C. (2000, February). El abordaje de la fobia a volar mediante realidad virtual (Approach to flying phobia by virtual reality). Communication presented at the IX Meeting of 
the Psychiatric Association of the Comunidad Valenciana, Castellón, Spain.

16. Botella, C., Villa, H., Baños, R., Perpiñá, C., and García-Palacios, A. (1999). The treatment of claustrophobia with virtual reality: Changes in other phobic behaviors not specifically treated. Cyberpsychology and Behavior, 2, 135-141.

17. Marco, J.H. (2000). Tratamiento de las alteraciones de la imagen corporal en los trastornos alimentarios: Eficacia diferencial de las técnicas de realidad virtual frente a las técnicas tradicionales (Treatment of body image disturbances in eating disorders: Differential efficacy of virtual reality techniques and traditional techniques). Unpublished master's thesis, Universidad Jaume I, Castellón, Spain.

18. Perpiñá, C., Botella, C., Baños, R., Marco, J.H, Alcañiz, M., and Quero, S. (1999). Body Image and virtual reality in eating disorders: Is exposure by virtual reality more effective than the classical body image treatment? Cyberpsychology and Behavior, 2, 149-159.

19. Glasgow, R., and Rosen, G. (1978). Behavioral bibliotherapy: A review of self-help behavior therapy manuals. Psychological Bulletin, 85, 1-23.

20. Glasgow, R., and Rosen, G. (1982). Self-help behavior therapy manuals: Recent development and clinical usage. Clinical Behavior Therapy Review, 1, 1-20.

21. Rosen, G.M. (1987). Self-treatment books and the commercialization of psychotherapy. American Psychologist, 42, 46-51.

22. Gould, R.A., and Clum, G.A. (1993). A meta-analysis of self-help treatment approaches. Clinical Psychology Review, 13, 169-186.

23. Botella, C., and García-Palacios, A. (1999). The possibility of reducing therapist contact and total length of therapy in the treatment of panic disorder. Behavioral and Cognitive Psychotherapy, 27, 231-247.

24. García-Palacios, A., and Botella, C. (1998). La interferencia de la utilización concurrente del alprazolam en el tratamiento cognitivo-comportamental del trastorno de pánico (Interference of the concurrent use of alprazolam in the cognitive-behavioral treatment of panic disorder). Revista de Psicopatología y Psicología Clínica, 3, 81-93.

25. Walker, J.R., Ross, C.A., and Norton, G.R. (1991). A final word: Pragmatic considerations for the practitioner. In: Walker, J.R., Norton, G.R., and Ross, C.A. (Eds.). Panic disorder and agoraphobia: A comprehensive guide for the practitioner. Pacific Grove, CA: Brooks/Cole.

26. Standing Committee of Family and Community Affairs. (1997). Health on line: A report on health information management and telemedicine. House of Representatives Parliament of the Commonwealth of Australia. Australian Government Publishing Service: Canberra, Australia.

27. Hill, S. (1997). Lessons from the Australian experience: Stage one interim report of the telemedicine evaluation project. Centre for the Study of Clinical Practice, St. Vincent's Hospital.

28. Banshur, R.L. (1995). Perspective: On the definition and evaluation of telemedicine. Telemedicine Journal, 1, 19-30.

29. Brown, F.W. (1998). Rural telepsychiatry. Psychiatric Services, 49, 963-964.

30. Baer, L., Cukor, P., and Coyle, J.T. (1997). Telepsychiatry: Application of telemedicine to psychiatry. In: Bashshur, R.L., and Sanders, J.J. (Eds.). Telemedicine: Theory and practice. Springfield: Charles C. Thomas, Publisher.

31. Kalucy, R.S. (1998). Telepsychiatry in South Australia. Journal of Telemedicine and Telecare, 4, 187-194.

32. Zajtchuk, R., and Gilbert, G.R. (1999). Telemedicine: A new dimension in the practice of medicine. Telemedicine, 45, 197-262.

33. Graham, M.A. (1996). Telepsychiatry in Appalachia. American Behavioral Scientist, 39, 602-615.

34. Harvey, R.J., Roques, P.K., Fox, N.C., and Rosor, M.N. (1998). CANDID: Counseling and diagnosis in dementia: A national telemedicine service supporting the care of younger patients with dementia. International Journal of Geriatric Psychiatry, 13, 381-388.

35. Bear, D., Jacobson, G., Aaronson, S., and Hanson, A. (1997). Telemedicine in psychiatry: Making the dream reality. American Journal of Psychiatry, 154, 884-885.

36. Blackmon, L.A., Kaak, H.O., and Ranseen, J. (1997). Consumer satisfaction with telemedicine child psychiatry consultation in rural Kentucky. Psychiatric Services, 48, 1464-1466.

37. Ermer, D.J. (1999). Experience with a rural telepsyquiatry clinic for children and adolescents. Psychiatric Services, 50, 260-261.

38. Jacobs, P. (1999). Evaluation of a telepsychiatry pilot project. Journal of Telemedicine and Telecare, 5, 38-46.

39. Stiers, W.M., and Kewman, D.G. (1997). Psychology and medical rehabilitation: Moving toward a consumer-driven health care system. Journal of Clinical Psychology in Medical Settings, 4, 167-179.

40. Rappaport, J. (1977). Community psychology: Values, research, and action. New York: Holt, Rinehart, and Winston.

41. DeLeon, J.L., James, P., and Gedney, J. (2000). The coming of age of telecommunications in psychological research and practice. American Psychologist, 55, 77-90.

42. Grohol, J.M. (1999). The insider's guide to mental health resources online. New York: Guilford Press.

43. Clum, G.A. (1990). Coping with panic. Pacific Grove, CA: Brooks /Cole Publishing.

Address reprint requests to: Cristina Botella, Ph.D. Universidad Jaume I de Castellón Facultad de Psicología Ctra. Borriol S/A 1208 Castellón, Spain

E-mail: botella@psb.uji.es 
Copyright of CyberPsychology \& Behavior is the property of Mary Ann Liebert, Inc. and its content may not be copied or emailed to multiple sites or posted to a listserv without the copyright holder's express written permission. However, users may print, download, or email articles for individual use. 